\title{
A GENERAL APPROACH FOR CALCULATING COUPLING IMPEDANCES OF SMALL DISCONTINUITIES
}

\author{
Sergey S. Kurennoy and Robert L. Gluckstern \\ Physics Department, University of Maryland, College Park, MD 20742, USA \\ and Gennady V. Stupakov \\ SLAC, Stanford, CA 94309, USA
}

\begin{abstract}
A general theory of the beam interaction with small discontinuities of the vacuum chamber is developed taking into account the reaction of radiated waves back on the discontinuity. The reactive impedance calculated earlier is reproduced as the first order, and the resistive one as the second order of a perturbation theory based on this general approach. The theory also gives, in a very natural way, the results for the trapped modes due to small discontinuities obtained earlier by a different method.
\end{abstract}

\section{Introduction}

A common tendency in design of modern accelerators is to minimize beam-chamber coupling impedances to avoid beam instabilities and reduce heating. Even contributions from tiny discontinuities like pumping holes have to be accounted for, due to their large number, which makes analytical methods for calculating the impedances of small discontinuities very important. According to the Bethe theory of diffraction by small holes [1], the fields diffracted by a hole can be found as those radiated by effective electric and magnetic dipoles. The coupling impedance of pumping holes in the vacuum chamber walls has been calculated earlier [2], [3], [4] using this idea. The imaginary part of the impedance is proportional to the difference of hole polarizabilities $(\psi-\chi)$, where the magnetic susceptibility $\psi$ and the electric polarizability $\chi$ are small compared to the cubed typical dimension $b^{3}$ of the chamber cross section. From considerations of the energy radiated into the chamber and through the hole, the real part of the hole impedance comes out to be proportional to $\left(\psi^{2}+\chi^{2}\right)$, being usually much smaller than the reactance.

In the present paper we further develop this analytical approach by taking into account the reaction of radiated waves back on the discontinuity. It leads to a more general theory, and allows us to reproduce easily all previous results, including those about trapped modes due to small discontinuities [5]. While our consideration here is restricted to small holes, it can be readily applied to other small discontinuities like enlargements or irises because the idea of effective polarizabilities works equally well in these cases also [6].

\section{General Analysis}

Let us consider an infinite cylindrical pipe with an arbitrary cross section $S$ and perfectly conducting walls. The $z$ axis is directed along the pipe axis, a hole is located at the point $(\vec{b}, z=$ 0 ), and a typical hole size $h$ satisfies $h \ll b$. To evaluate the coupling impedance one has to calculate the fields induced in the chamber by a given current. If an ultrarelativistic point charge $q$ moves along the chamber axis, the fields harmonics $\vec{E}^{b}, \vec{H}^{b}$ produced by this charge on the chamber wall without hole would be

$$
E_{\nu}^{b}(z ; \omega)=Z_{0} H_{\tau}^{b}=-Z_{0} q e^{i k z} \sum_{n, m} \frac{e_{n m}(0) \nabla_{\nu} e_{n m}(\vec{b})}{k_{n m}^{2}},
$$

where $k_{n m}^{2}, e_{n m}(\vec{r})$ are eigenvalues and orthonormalized eigenfunctions (EFs) of the 2D boundary problem in $S$ :

$$
\left(\nabla^{2}+k_{n m}^{2}\right) e_{n m}=0 ;\left.e_{n m}\right|_{\partial S}=0 .
$$

Here $\vec{\nabla}$ is the 2D gradient in plane $S ; k=\omega / c ; \hat{v}$ means an outward normal unit vector, $\hat{\tau}$ is a unit vector tangent to the boundary $\partial S$ of the chamber cross section $S$, and $\{\hat{v}, \hat{\tau}, \hat{z}\}$ form a RHS basis.

\section{A. Fields}

At distances $l$ such that $h \ll l \ll b$, the fields radiated by the hole into the pipe are equal to those produced by effective dipoles [1], [7] ${ }^{1}$

$$
\begin{aligned}
P_{v} & =-\chi \varepsilon_{0} E_{v}^{h} / 2 ; \quad M_{\tau}=\left(\psi_{\tau \tau} H_{\tau}^{h}+\psi_{\tau z} H_{z}^{h}\right) / 2 ; \\
M_{z} & =\left(\psi_{z \tau} H_{\tau}^{h}+\psi_{z z} H_{z}^{h}\right) / 2,
\end{aligned}
$$

where superscript ' $h$ ' means that the fields are taken at the hole. In general, $\psi$ is a symmetric 2D-tensor. If the hole is symmetric, and its symmetry axis is parallel to $\hat{z}$, the skew terms vanish, i.e. $\psi_{\tau z}=\psi_{z \tau}=0$.

When the effective dipoles are obtained, e.g., by substituting beam fields (1) into Eqs. (3), one can calculate the fields in the chamber as a sum of waveguide eigenmodes excited in the chamber by the dipoles, and find the impedance. This approach has been carried out in [2], and for an arbitrary chamber in [8]. However, a more refined theory should take into account the reaction of radiated waves back on the hole. The TM-eigenmodes contribution to the radiated fields is a series

$$
\vec{F}=\sum_{n m}\left[A_{n m}^{+} \vec{F}_{n m}^{+} \theta(z)+A_{n m}^{-} \vec{F}_{n m}^{-} \theta(-z)\right],
$$

where $\vec{F}$ means either $\vec{E}$ or $\vec{H}$ and superscripts ' \pm ' denote waves radiated respectively in the positive $(+, z>0)$ or negative $(-$, $z<0$ ) direction. The fields of $\{n, m\}$ th TM-eigenmode in Eq. (4)

\footnotetext{
${ }^{1}$ Polarizabilities $\psi, \chi$ are related to the effective ones $\alpha_{e}, \alpha_{m}$ used in [7], [2] as $\alpha_{e}=-\chi / 2$ and $\alpha_{m}=\psi / 2$, so that for a circular hole of radius $a$ in a thin wall $\psi=8 a^{3} / 3$ and $\chi=4 a^{3} / 3$.
} 
are expressed [7] in terms of EFs (2)

$$
\begin{aligned}
E_{z}^{\mp} & =k_{n m}^{2} e_{n m} \exp \left( \pm \Gamma_{n m} z\right) ; \quad H_{z}^{\mp}=0 ; \\
\vec{E}_{t}^{\mp} & = \pm \Gamma_{n m} \vec{\nabla} e_{n m} \exp \left( \pm \Gamma_{n m} z\right) ; \\
\vec{H}_{t}^{\mp} & =\frac{i k}{Z_{0}} \hat{z} \times \vec{\nabla} e_{n m} \exp \left( \pm \Gamma_{n m} z\right),
\end{aligned}
$$

where propagation factors $\Gamma_{n m}=\left(k_{n m}^{2}-k^{2}\right)^{1 / 2}$ should be replaced by $-i \beta_{n m}$ with $\beta_{n m}=\left(k^{2}-k_{n m}^{2}\right)^{1 / 2}$ for $k>k_{n m}$. For given values of dipoles (3) the unknown coefficients $A_{\mathrm{nm}}^{ \pm}$can be found [8] using the Lorentz reciprocity theorem

$$
\begin{gathered}
A_{n m}^{ \pm}=a_{n m} M_{\tau} \pm b_{n m} P_{\nu} \\
a_{n m}=-\frac{i k Z_{0}}{2 \Gamma_{n m} k_{n m}^{2}} \nabla_{\nu} e_{n m}^{h} ; \quad b_{n m}=\frac{1}{2 \varepsilon_{0} k_{n m}^{2}} \nabla_{\nu} e_{n m}^{h} .
\end{gathered}
$$

In a similar way, the contribution of TE-eigenmodes to the radiated fields is given by an analogue of Eq. (4) with the excitation coefficients

$$
\begin{gathered}
B_{n m}^{ \pm}= \pm c_{n m} M_{\tau}+d_{n m} P_{v}+q_{n m} M_{z}, \\
c_{n m}=\frac{1}{2 k_{n m}^{\prime 2}} \nabla_{\tau} h_{n m}^{h} ; \quad q_{n m}=\frac{1}{2 \Gamma_{n m}^{\prime}} h_{n m}^{h} ; \\
d_{n m}=-\frac{i k}{2 Z_{0} \varepsilon_{0} \Gamma_{n m}^{\prime} k_{n m}^{\prime 2}} \nabla_{\tau} h_{n m}^{h},
\end{gathered}
$$

where EFs $h_{n m}$ satisfy the boundary problem (2) with the Neumann boundary condition $\left.\nabla_{\nu} h_{n m}\right|_{\partial S}=0$, and $k_{n m}^{\prime 2}$ are corresponding eigenvalues.

Now we can add corrections to the beam fields (1) due to the radiated waves in the vicinity of the hole. It gives

$$
\begin{aligned}
E_{v} & =\frac{E_{v}^{b}+\psi_{z \tau} \Sigma_{x}^{\prime} Z_{0} H_{\tau}+\psi_{z z} \Sigma_{x}^{\prime} Z_{0} H_{z}}{1-\chi\left(\Sigma_{1}-\Sigma_{1}^{\prime}\right)}, \\
H_{\tau} & =\frac{H_{\tau}^{b}+\psi_{\tau z}\left(\Sigma_{2}-\Sigma_{2}^{\prime}\right) H_{z}}{1-\psi_{\tau \tau}\left(\Sigma_{2}-\Sigma_{2}^{\prime}\right)}, \\
H_{z} & =\frac{\chi \Sigma_{x}^{\prime} E_{v} / Z_{0}+\psi_{z \tau} \Sigma_{3}^{\prime} H_{\tau}}{1-\psi_{z z} \Sigma_{3}^{\prime}},
\end{aligned}
$$

where $(s=\{n, m\}$ is a generalized index $)$

$$
\begin{aligned}
& \Sigma_{1}=\frac{1}{4} \sum_{s} \frac{\Gamma_{s}\left(\nabla_{\nu} e_{s}^{h}\right)^{2}}{k_{s}^{2}} ; \Sigma_{2}=\frac{k^{2}}{4} \sum_{s} \frac{\left(\nabla_{\nu} e_{s}^{h}\right)^{2}}{\Gamma_{s} k_{s}^{2}} ; \\
& \Sigma_{1}^{\prime}=\frac{k^{2}}{4} \sum_{s} \frac{\left(\nabla_{\tau} h_{s}^{h}\right)^{2}}{\Gamma_{s}^{\prime} k_{s}^{\prime 2}} ; \Sigma_{2}^{\prime}=\frac{1}{4} \sum_{s} \frac{\Gamma_{s}^{\prime}\left(\nabla_{\tau} h_{s}^{h}\right)^{2}}{k_{s}^{\prime 2}} ; \\
& \Sigma_{x}^{\prime}=i \frac{k}{4} \sum_{s} \frac{h_{s}^{h} \nabla_{\tau} h_{s}^{h}}{\Gamma_{s}^{\prime}} ; \Sigma_{3}^{\prime}=\frac{1}{4} \sum_{s} \frac{k_{s}^{\prime 2}\left(h_{s}^{h}\right)^{2}}{\Gamma_{s}^{\prime}} .
\end{aligned}
$$

Since this consideration works at distances not shorter than $l$, and $l>h$, the summation in Eq. (13) should be restricted to values of $s=\{n, m\}$ such that $k_{s} h \leq 1$ and $k_{s}^{\prime} h \leq 1$.

\section{B. Impedance}

The longitudinal impedance of the hole is defined as

$$
Z(k)=-\frac{1}{q} \int_{-\infty}^{\infty} d z e^{-i k z} E_{z}(0, z ; \omega),
$$

where the field at the axis is given by Eq. (4) with coefficients (6) and (8) in which the corrected near-hole fields (10)-(12) are substituted. It yields

$$
\begin{aligned}
Z(k)= & -\frac{i k Z_{0} \tilde{e}_{v}^{2}}{2}\left[\frac{\psi_{\tau \tau}}{1-\psi_{\tau \tau}\left(\Sigma_{2}-\Sigma_{2}^{\prime}\right)}\right. \\
& \left.+\psi_{\tau z}^{2} \Sigma_{3}^{\prime}-\frac{\chi}{1-\chi\left(\Sigma_{1}-\Sigma_{1}^{\prime}\right)}\right],
\end{aligned}
$$

where $\tilde{e}_{v} \equiv E_{v}^{b} /\left(Z_{0} q\right)=-\sum_{s} e_{s}(0) \nabla_{\nu} e_{s}(\vec{b}) / k_{s}^{2}$ is merely the normalized electric field produced at the hole location by the beam moving along the chamber axis, cf. Eq. (1). In deriving this result we have neglected the coupling terms between $E_{\nu}, H_{\tau}$ and $H_{z}$, cf. Eqs. (10)-(12), which contribute to the third order of an expansion discussed below, and also have taken into account that $\psi_{\tau z}=\psi_{z \tau}$.

For a small discontinuity, polarizabilities $\psi, \chi=O\left(h^{3}\right)$, and they are small compared to $b^{3}$. If we expand the impedance (15) in a perturbation series in polarizabilities, the first order gives

$$
Z_{1}(k)=-\frac{i k Z_{0} \tilde{e}_{v}^{2}}{2}\left(\psi_{\tau \tau}-\chi\right),
$$

that is exactly the inductive impedance obtained in [8] for an arbitrary cross section of the chamber. For a particular case of a circular pipe, from either direct summation in (1) or applying the Gauss law, we get $\tilde{e}_{v}=1 /(2 \pi b)$, substitution of which into Eq. (16) leads to a well-known result [2], [3]. From a physical point of view, keeping only the first order term (16) corresponds to dropping out all radiation corrections in Eqs. (10)-(12).

These corrections first reveal themselves in the second order term

$$
\begin{array}{r}
Z_{2}(k)=-\frac{i k Z_{0} \tilde{e}_{v}^{2}}{2}\left[\psi_{\tau \tau}^{2}\left(\Sigma_{2}-\Sigma_{2}^{\prime}\right)+\psi_{\tau z}^{2} \Sigma_{3}^{\prime}\right. \\
\left.+\chi^{2}\left(\Sigma_{1}^{\prime}-\Sigma_{1}\right)\right]
\end{array}
$$

which at frequencies above the chamber cutoff has both a real and imaginary part. The real part of the impedance is

$$
\begin{aligned}
\operatorname{ReZ}_{2}(k) & =\frac{k^{3} Z_{0} \tilde{e}_{v}^{2}}{8}\left\{\psi_{\tau z}^{2} \sum_{s}^{<} \frac{k_{s}^{\prime 2}\left(h_{s}^{h}\right)^{2}}{k^{2} \beta_{s}^{\prime}}\right. \\
& +\psi_{\tau \tau}^{2}\left[\sum_{s}^{<} \frac{\left(\nabla_{\nu} e_{s}^{h}\right)^{2}}{\beta_{s} k_{s}^{2}}+\sum_{s}^{<} \frac{\beta_{s}^{\prime}\left(\nabla_{\tau} h_{s}^{h}\right)^{2}}{k^{2} k_{s}^{\prime 2}}\right] \\
& \left.+\chi^{2}\left[\sum_{s}^{<} \frac{\beta_{s}\left(\nabla_{\nu} e_{s}^{h}\right)^{2}}{k^{2} k_{s}^{2}}+\sum_{s}^{<} \frac{\left(\nabla_{\tau} h_{s}^{h}\right)^{2}}{\beta_{s}^{\prime} k_{s}^{\prime 2}}\right]\right\},
\end{aligned}
$$

where the sums include only a finite number of the eigenmodes propagating in the chamber at a given frequency, i.e. those with $k_{s}<k$ or $k_{s}^{\prime}<k$.

The real part of the impedance is related to the power $P$ scattered by the hole into the beam pipe, $\operatorname{Re} Z=2 P / q^{2}$, and can 
be calculated in an alternative way from energy considerations: $P=\sum_{s}\left(A_{s}^{2} P_{s}^{(E)}+B_{s}^{2} P_{s}^{(H)}\right)$, where we sum over all propagating modes in both directions, and $P_{s}$ means the time-averaged power radiated in $s$ th eigenmode: $P_{s}^{(E)}=k \beta_{s} k_{s}^{2} /\left(2 Z_{0}\right)$ and $P_{s}^{(H)}=Z_{0} k \beta_{s}^{\prime} k_{s}^{\prime 2} / 2$. Substituting beam fields (1) into Eqs. (6)(9) for the coefficients $A_{s}$ and $B_{s}$ and performing calculations gives the result (18). Such an alternative derivation of the real part has been first carried out in Ref. [4] for a circular pipe with a symmetric untilted hole $\left(\psi_{\tau z}=0\right)$. The result (18) for this particular case coincides with that of [4]. Moreover, in this case at high frequencies the series can be summed approximately [4] to give $\operatorname{Re} Z=Z_{0} k^{4} \tilde{e}_{v}^{2}\left(\psi_{\tau \tau}^{2}+\chi^{2}\right) /(12 \pi)$, which can also be obtained by calculating the energy radiated by the dipoles in a half-space [8]. Note that the additional $\psi_{\tau z}^{2}$-term in Eq. (18) could give a leading contribution to $R e Z$, e.g., for a long and slightly tilted slot.

\section{Trapped Modes}

So far we considered the perturbation expansion of Eq. (15) implicitly assuming that correction terms $O(\psi)$ and $O(\chi)$ in the denominators of its RHS are small compared to 1 . Under certain conditions this assumption is incorrect, and it leads to some non-perturbative results. Indeed, at frequencies slightly below the chamber cut-offs, $0<k_{s}-k \ll k_{s}$, - or the same with replacement $k_{s} \rightarrow k_{s}^{\prime}$, - a single term in sums $\Sigma_{1}^{\prime}, \Sigma_{2}$, or $\Sigma_{3}^{\prime}$ becomes very large, due to very small $\Gamma_{s}=\left(k_{s}^{2}-k^{2}\right)^{1 / 2}$ (or $\Gamma_{s}^{\prime}$ ) in its denominator, and then the "corrections" $\psi \Sigma$ or $\chi \Sigma$ can be of the order of 1 . As a result, one of the denominators of the RHS of Eqs. (15) can vanish, which corresponds to a resonance of the coupling impedance. On the other hand, vanishing denominators in Eqs. (10)-(12) mean the existence of non-perturbative eigenmodes of the chamber with a hole, since non-trivial solutions $E, H \neq 0$ exist even for vanishing external (beam) fields $E^{b}, H^{b}=0$. These eigenmodes are nothing but the trapped modes studied in [5] for a circular waveguide with a small discontinuity (see [9] for waveguides with an arbitrary cross section).

Let us for brevity restrict ourselves to the case $\psi_{\tau z}=0$ and consider Eq. (11) in more detail. For $H^{b}=0$ we have

$$
H_{\tau}\left[1-\psi_{\tau \tau} \frac{k^{2}\left(\nabla_{\nu} e_{n m}^{h}\right)^{2}}{4 \Gamma_{n m} k_{n m}^{2}}+\ldots\right]=0,
$$

where ... means all other terms of the series $\Sigma_{2}, \Sigma_{2}^{\prime}$. At frequency $k \simeq k_{n m}$ slightly below the cutoff $k_{n m}$ of the $\mathrm{TM}_{n m}$-mode, the fraction in Eq. (19) is large due to small $\Gamma_{n m}$ in its denominator, and one can neglect the other terms. Then the condition for a non-trivial solution $H_{\tau} \neq 0$ to exist is

$$
\Gamma_{n m} \simeq \frac{1}{4} \psi_{\tau \tau}\left(\nabla_{\nu} e_{n m}^{h}\right)^{2}
$$

This equation gives us the frequency shift $\Delta f$ of the trapped TM-mode down from the cutoff $f_{n m}^{(E)}$

$$
\frac{\Delta f}{f_{n m}^{(E)}} \simeq \frac{1}{32 k_{n m}^{2}} \psi_{\tau \tau}^{2}\left(\nabla_{\nu} e_{n m}^{h}\right)^{4} .
$$

One can easily see that denominator $\left[1-\chi\left(\Sigma_{1}-\Sigma_{1}^{\prime}\right)\right]$ in Eq. (10) does not vanish because singular terms in $\Sigma_{1}^{\prime}$ have a "wrong" sign. However, due to the coupling between $E_{v}$ and $H_{z}$, a non-trivial solution $E_{v}, H_{z} \neq 0$ of simultaneous equations (10) and (12) can exist, even when $E^{b}=0$. The corresponding condition has the form

$$
\Gamma_{n m}^{\prime} \simeq \frac{1}{4}\left[\psi_{z z} k_{n m}^{\prime 2}\left(h_{n m}^{h}\right)^{2}-\chi\left(\nabla_{\tau} h_{n m}^{h}\right)^{2}\right]
$$

which gives the frequency of the trapped $\mathrm{TE}_{n m}$-mode.

One can easily show that for the particular case of a circular pipe the results (20)-(22) coincide with those obtained by a different method in Ref. [5]. For more detail, a physical picture of, and resonance impedances due to trapped modes, see [5] and [9].

\section{Conclusions}

The analytical approach discussed above provides a general picture for the coupling impedance of a small discontinuity of the vacuum chamber. It gives the real and imaginary part of the impedance, as well as trapped modes. Results for typical shapes of the chamber cross section (circular or rectangular) are easily obtained from the expressions above using specific EFs, see, e.g., in [7] or [9]. The transverse impedance can be derived in a similar way [8].

We have not considered explicitly effects of the wall thickness, assuming that the hole polarizabilities are the inside ones [3], and they include these effects. We also neglected the radiation escaping through the hole, contributions of which to the real part of the impedance are estimated [2], [3], [5], and usually are small.

At high frequencies (near or above the chamber cutoff) the mutual interaction of many holes is important and can cause resonances if the hole pattern is periodic, e.g. [10], [4]. A more complete theory should take this interaction into account.

\section{References}

[1] H.A. Bethe, Phys. Rev. 66 (1944) 163.

[2] S.S. Kurennoy, Part. Acc. 39 (1992) 1.

[3] R.L. Gluckstern, Phys. Rev. A 46 (1992) 1106, 1110.

[4] G.V. Stupakov, Preprint SLAC-6698, Stanford (1994); Phys. Rev. E, to be published.

[5] G.V. Stupakov and S.S. Kurennoy, Phys. Rev. E 49 (1994) 794.

[6] S.S. Kurennoy and G.V. Stupakov, Part. Acc. 45 (1994) 95.

[7] R.E. Collin, Field Theory of Guided Waves (IEEE, NY, 1991).

[8] S.S. Kurennoy, Proceed. of EPAC (Berlin, 1992) 871; more details in IHEP 92-84, Protvino (1992).

[9] S.S. Kurennoy and G.V. Stupakov, These Proceedings.

[10] S.S. Kurennoy, Proceed. of PAC (Washington, DC, 1993) 3417. 